\title{
EL DESCENTRAMIENTO DEL AGENTE TÉCNICO \\ UNA CONFRONTACIÓN DE LA FILOSOFÍA DEL PRIMER HEIDEGGER CON LA FILOSOFÍA DE LA TÉCNICA DE G. SIMONDON
}

\author{
THE OFF-CENTERING OF THE TECHNICAL AGENT \\ A CONFRONTATION BETWEEN HEIDEGGER'S EARLY PHILOSOPHY AND G. \\ SIMONDON'S PHILOSOPHY OF TECHNOLOGY
}

\begin{abstract}
LUCIANO MASCARÓ
lcnmascaro@hotmail.com

Consejo Nacional de Investigaciones Científicas y Técnicas - Centro de estudios Filosóficos de la Academia Nacional de Ciencias de Buenos Aires - Universidad de Buenos Aires (Argentina)
\end{abstract}

RECIBIDO: $12 / 02 / 2019$
ACEPTADO: 01/04/2019

Resumen: Esta investigación se propone realizar una breve revisión de la ontología de los objetos técnicos que se encuentra presente en Ser y Tiempo. Intentaremos mostrar que, aunque los objetivos de esta obra no se dirigían a la elucidación de la estructura de los artefactos, sería posible extraer una suerte de filosofía de la técnica de corte existencial, pero, para que esta filosofía sea capaz de dialogar con las corrientes contemporáneas sería necesario expandirla para superar una serie de limitaciones. Consideramos que esta ampliación y actualización podría llevarse a cabo por medio de un diálogo con la filosofía de G. Simondon. El resultado de este intercambio sería un descentramiento de la existencia humana en la escena técnica.

Palabras clave: Heidegger, Simondon, tecnología, objeto técnico, mundo, espacio, materia.

\begin{abstract}
This research attempts to perform a brief review of the ontology of technical objects, as it is showcased in M. Heidegger's Sein und Zeit. We will seek to show that, even though the goals of this work did not aim to elucidate the structure of artifacts, it would be possible to extract a form of existential hpilosophy of technology; however, in order for this philosophy to be capable of engaging dialogue with contemporary currents of thought, it would be necesary to expand it, to make it able to overcome a few limitations. We believe that this amplification and updating could be carried out via a dialogue with G.Simondon's philosophy. The result of this Exchange would be an off-centering of human existence in te technological scene.
\end{abstract}

Keywords: Heidegger, Simondon, Technology, technical object, World, space, matter

\section{Sobre la delimitación de las obras analizadas:}

En la presente investigación no nos ocuparemos de las obras heideggerianas posteriores a la Kehre de los años '30. Esta opción puede parecer sorprendente, 
dado que nos proponemos pensar el problema de la ontología de los artefactos, y es justamente en el segundo período del pensamiento de Heidegger donde la cuestión de la técnica se vuelve una temática central y explícita. En el presente análisis nos centraremos en la obra fundamental del primer período del pensamiento de Heidegger, Ser y Tiempo, como escrito culminante de su filosofía de los años'20; esta delimitación responde a varios motivos: En primer lugar, nos concentraremos en el período mencionado puesto que el mismo suele quedar relegado en los estudios acerca del problema de la técnica en Heidegger. En las lecciones tempranas de Friburgo (donde se reinterpreta la téchne aristotélica) y en los escritos de la Kehre y posteriores, la cuestión de la técnica parece cobrar una relevancia central, por ello los especialistas tienden a dirigir su atención sobre estas obras. Con esta situación en vista, intentaremos reconquistar -por medio de una obra fundamental- un período postergado en las investigaciones, pero de una enorme riqueza en lo referente al estudio de la estructura de los artefactos. En segundo lugar, consideramos que la primacía del segundo Heidegger en los estudios acerca de la técnica responde a un interés interpretativo que nosotros no acompañaremos: el Heidegger de los años '30 y posteriores no se interesa en verdad por el modo de existencia de los objetos técnicos, sino por la elucidación de la esencia de la técnica como momento culminante en la historia del olvido del ser. El segundo Heidegger sólo menciona una serie de complejos técnicos, pero no analiza los verdaderos procesos que ocurren en su interior, como si éstos fuesen de común conocimiento. El interés del segundo Heidegger no está dirigido a la elucidación de los encadenamientos físicos, causales y operativos de los entes técnicos o de los sistemas industriales (lo que en este trabajo nos interesa), sino, según él mismo lo indica, al descubrimiento de la esencia de la técnica; y dado que "la esencia de la técnica no es nada técnico" (Heidegger 1994:9) y que la concentración reflexiva en los artefactos, máquinas y sistemas constituirían un desvío o resignación ante una cuestión más urgente, difícilmente encontraremos en su pensamiento posterior a la Kehre una descripción del modo en que los complejos industriales llegan a desdibujar los límites del mundo circundante. El segundo Heidegger suspende el análisis óntico de los objetos técnicos, es decir, ya no estudia los artefactos desde ellos mismos, en busca de su estructura, es por ello que no podremos seguirlo para aportar claridad a la cuestión que nos interesa.

\section{Introducción}

Esta investigación surge del estudio de temáticas y autores asociados a la contemporánea filosofía de la tecnología, la cual se realiza desde la perspectiva y 
el horizonte establecido por nuestra especialización en M.Heidegger. En esta aproximación se detectaron una serie de asuntos que condujeron a cuestionamientos que son los que movilizan nuestra investigación actual en general, y este trabajo en particular. Las cuestiones que mencionamos a continuación constituyen el disparador para el trabajo que aquí se intenta. Los interrogantes que analizaremos en la presente investigación tomaron forma en una serie sucesiva de pasos. En esta oportunidad no podremos fundamentar todas las consideraciones preparatorias, puesto que ellas se arraigan en estudios previos de nuestra autoría:

1) En primer lugar, llamó nuestra atención el hecho de que la amplia mayoría de los trabajos que estudian el problema de la técnica en Heidegger se centran en su filosofía posterior a los años ' 30 . Pocos estudian su tratamiento de los objetos técnicos en las lecciones de los años ' 20.

2) Pudimos constatar que la filosofía del Heidegger posterior a la Kehre de los años ' 30 en verdad no estudia la estructura interna y el funcionamiento de artefactos, máquinas o sistemas técnicos, sino que sólo los menciona. El objetivo de la filosofía heideggeriana posterior a los '30 es superar este nivel óntico de análisis, en busca de la esencia de la técnica, en tanto Gestell, provocación, y última etapa en la historia del olvido del ser, y culminación de la metafísica occidental. Dado que nuestro interés se dirige a articular las reflexiones acerca e la estructura ontológica de las herramientas, en la presente investigación, no se atenderá a las obras del segundo Heidegger.

3) También pudimos constatar que en el primer período del pensar de Heidegger existe una investigación mucho más minuciosa de herramientas concretas ${ }^{1}$. Es en el primer período de su pensamiento donde, a nuestro parecer, se encuentran los verdaderos aportes heideggerianos a la filosofía de la tecnología -si por ella entendemos un análisis de la estructura ontológica de los objetos técnicos- (esta opinión es compartida por Walther Zimmerli, Bernhardt Irrgang, y de Don Ihde, entre otros). Desde luego, este estudio de artefactos no constituye en modo alguno el objetivo final de las reflexiones de Ser y Tiempo y otros textos del período, sino que se inscribe en un proyecto mucho mayor y de dimensiones ontológicas: la elucidación de la constitución existencial del Dasein, como punto de acontecimiento del fenómeno del Sentido, y de la pregunta por el ser. Con todo, este direccionamiento de las reflexiones heideggerianas no nos impide a nosotros tomar los elementos más relevantes de su análisis de herramientas, para integrarlos y actualizarlos en una ontología de los artefactos

\footnotetext{
${ }^{1}$ interpretadas desde el modelo de la herramienta, y no, por ejemplo, desde el modelo de la obra de arte, como es el caso la jarra en la conferencia "la cosa"
} 
de corte heideggeriano, capaz de dialogar con las corrientes contemporáneas que se ocupan del estudio de las herramientas y las máquinas. Pues bien, ante esta situación, surgió la pregunta:

4) ¿se podría extraer del proyecto ontológico de Ser y Tiempo (y de la filosofía del período de Marburgo en general) una filosofía de los entes técnicos (das zuhandene)?

5) Si esto fuese posible ¿cuáles serían las limitaciones de esta filosofía? Nuestra intuición fue que las limitaciones consistirían en la preeminencia del modelo artesanal de la técnica, la primacía del esquema hylemórfico y funcionalista a la hora de realizar explicaciones, y la falta de estudio del funcionamiento de artefactos y máquinas concretas. Por tanto, si deseamos que la filosofía de la técnica del primer Heidegger pueda dialogar con problemáticas tecnológicas contemporáneas, deberíamos realizar una suerte de ampliación y actualización de su armazón conceptual.

6) El último paso de nuestro trayecto fue el intento de demostrar una intuición: que esa actualización y ampliación podría ser llevada a cabo por medio de un diálogo con la filosofía de G.Simondon, un filósofo arraigado en la escena técnica contemporánea, y autor de sutiles análisis ontológicos sobre la estructura y funcionamiento máquinas concretas. Un filósofo que, según él mismo afirma, también considera que el estudio de las actividades técnicas humanas y las relaciones humano-herramienta deben partir de un análisis de la existencia (Simondon, 2007:244)

7) En este punto inicia el trabajo que ahora presentamos: aquí se intentará mostrar que un estudio de corte heideggeriano que tome en cuenta -de la mano de un filósofo contemporáneo de la técnica- la complejidad de los sistemas técnicos actuales, y el modo en el que las máquinas son capaces de portar herramientas, organizar el espacio, y encarnar causalidades físicas y químicas, obtendría como resultado un cierto descentramiento de la figura del Dasein como organizador de la escena técnica y como polo de estructuración del sentido de las herramientas y máquinas.

Como es sabido, en el contexto de la filosofía del primer Heidegger, el mundo funciona como una estructura reticular de sentidos inicialmente pragmáticos interconectados. El conjunto remisional obtiene su sentido y dirección pragmática del proyecto fáctico que desarrolla un ente que se ubica en su centro: la existencia humana en tanto Worumwillen. El concepto de Worumwillen le otorga al Dasein un papel central en la configuración del mundo del trabajo: es su interés y su proyecto fáctico y técnico el que determinará la disposición de los artefactos, de los espacios, y de las conexiones funcionales entre las máquina y los usuarios. En otras palabras, el Dasein tiene un papel 
rector (aunque no manifiesto para sí mismo) en la configuración del medio técnico. Desde luego, el medio técnico siempre es público e histórico y no responde a la intención de un único organizador individual, sino a un colectivo de organizadores y actores. De cualquier modo, el papel del ser humano sigue siendo prioritario, $\mathrm{y}$, fundamentalmente, la esencia del artefacto sigue siendo comprendida en términos de funcionalidad, una postura que podría denominarse intencionalista (Parente y Crelier, 2015: 20 y ss.).

Con todo, la centralidad del Dasein parece insuficiente para explicar el punto de condensación de sentido del mundo técnico; en efecto, los mismos artefactos, y las causalidades físicas que en ellos operan traerán exigencias a la organización del medio técnico ante las cuales el Dasein sólo podrá adoptar un rol pasivo o adaptativo, si acaso desea que los procesos físicos y químicos de los que depende su actividad ocurran de hecho. Puntualmente, revisaremos cinco nociones que pueden provocar un descentramiento de la existencia humana como organizadora del mundo del trabajo: 1) el objeto técnico como portador de herramientas; 2) la crítica a la idea de funcionalidad como esencia del artefacto, en favor de la idea de gesto, linaje y genealogía de sinergias crecientes; 3) la capacidad del objeto técnico de diseñar el espacio del trabajo; 4) el objeto técnico como ámbito para el acontecimiento de procesos naturales; 5) el carácter obsoleto del modelo hylemórfico para la explicación de las tareas técnicas.

Por último aclaramos que en esta investigación realizaremos afirmaciones que pueden resultar algo confusas desde un punto de vista antropológico tales como "La máquina porta herramientas", "el objeto técnico configura espacio", "la materia trae exigencias", o "la máquina sugiere". No intentamos antropomorfizar los entes técnicos. Coincidimos con Heidegger en que sólo la existencia humana es capaz de portar, configurar, exigir y sugerir, ya que sólo ella se caracteriza como relación con el sentido del ser, o sea, sólo ella puede establecer vínculos significativos con los entes. Este modo de expresarnos busca enfatizar por medio de formulaciones donde el objeto técnico aparece como sujeto y no como objeto, la relevancia de la perspectiva que no considera la realidad técnica por referencia exclusiva a la participación humana en ella (perspectiva que consagra la primacía de la función), sino en tanto que estructurada en torno a una lógica propia, compuesta de causalidades, procesos, encadenamientos e interferencias, ensamblajes zonales y concurrencia de operaciones. Ante esta caracterización, la actitud de la existencia humana (la única capaz de vinculación significativa) frecuentemente se mostrará como adaptativa. Las expresiones que utilizamos no buscan transformar a los entes técnicos en seres capaces de comprensión, sino acentuar la falta de control por parte del agente humano de todas las fases del diseño del mundo del trabajo. 


\section{La noción de Mundo del trabajo y la Worumwillen}

Comenzaremos por explicitar algunas de las nociones fundamentales que, en nuestra opinión, podrían configurar una filosofía de los objetos técnicos de corte heideggeriano, y luego, analizaremos algunas limitaciones de las mismas.

La noción existencial de Mundo lo define como un entramado de relaciones significativas, una red de sentidos interdependientes en la cual cada ente o núcleo significativo dice referencia a los demás, y obtiene de ellos su sentido; cada vez, en función del proyectarse fáctico de una existencia. El mundo de la cotidianidad media, encuentra un tipo de ente paradigmático: el útil o artefacto [Zeug]. El uso de artefactos constituye la forma primaria de relación del ser humano con el mundo; para esta modalidad, el artefacto se define por su remisionalidad, todo artefacto "es para" $[U m-z u]$ y en esta finalidad, en este quedar referido al fin práctico, radica su esencia. La aproximación que destaca el papel fundamental de la existencia en el circuito de sentido de toda ocupación con útiles convierte a la remisionalidad del artefacto en condición respectiva [Bewandtnis]. En efecto, el entramado de remisiones obtiene su sentido de una serie de referencias interrelacionadas: martillo $\rightarrow$ martillar $\rightarrow$ reparar un tejado $\rightarrow$ evitar filtraciones de agua $\rightarrow$ permitir el habitar del Dasein. Como vemos, existe una remisión final de todas las condiciones respectivas: el denominado por mor de qué [Worumwillen ${ }^{2}$ ], que constituye el centro desde donde brota el sentido de la totalidad respeccional [Bewandtnisganzheit]. "Aquello hacia lo cual algo es dejado respeccionalmente en libertad es una totalidad respeccional, y últimamente, el Mundo, el cual ya debe estar pre-descubierto" (Tugendhat, 1970:290). La Worumwillen es la condición de posibilidad para que lo intramundano aparezca como a la mano. El mundo, en tanto entramado de entes y finalidades, articulados por relaciones de significado que se abren en función del proyectar del Dasein y que lo poseen a él como remisión final, queda caracterizado como significatividad [Bedeutsamkeit] (Heidegger, 2006:113).

La Worumwillen (también llamada Umwillen) nombra el hecho de que la existencia humana se tiene a sí misma (Heidegger, 2000:214), o bien, el hecho de que a ella le concierne (le va [geht um]) su propio ser, y por ello mismo, este ser no puede quedar en respectividad de otra cosa (Heidegger, 2007:368). La comprensión de la funcionalidad ${ }^{3}$ se funda en la comprensión del despliegue de los proyectos fácticos del Dasein: "Solo en la medida en que se comprende el

\footnotetext{
${ }^{2}$ Traducida como "por-mor-de-qué" por J.E. Rivera, y como "en-vista-de" por J.J. García Norro

${ }^{3}$ En este esquema, la esencia del artefacto, y característica determinante del mundo técnico
} 
"en-vista-de" [Umwillen] de un poder ser, se hace develable algo como el "para" [Um-zu] (relación de funcionalidad)" (Heidegger,2000:352)

El por-mor-de-qué nombra el proyectar, ya sea individual o grupal, pero siempre histórico y público de la existencia humana en el mundo, en tanto que ella motiva, ordena y orienta las conexiones significativas entre los entes y los límites del mundo circundante en función del quehacer ocupacional (en el caso del mundo del trabajo). Esta perspectiva, si bien no desconoce la independencia del artefacto de la instancia productora una vez que ha sido construido y la independencia funcional de los útiles, los ubica en un lugar de manifiesta subordinación significativa y organizativa con respecto al productor y usuario humano. Pero ¿qué pasaría si los objetos técnicos apareciesen revestidos de una serie de características que no dependiesen de la proyección del productor humano?¿qué lugar quedaría para el Dasein en un esquema del mundo circundante técnico que ya no lo reconoce como centro último de configuración de sentido y máximo referente de la proyección? ¿que pasaría si la independencia de los objetos técnicos se acentuase? ¿no obtendríamos un descentramiento de la figura del Dasein dentro del mundo del trabajo, una deflación de su papel ordenador y semantizante? Consideramos que la respuesta es afirmativa, e incluso que dicho descentramiento de la existencia es necesario si se desea facilitar el diálogo de la filosofía heideggeriana de los objetos técnicos con los debates contemporáneos, y si se busca comprender la realidad técnica actual como contrapuesta al modelo de producción artesanal, que es el que Heidegger prioriza en los estudios del primer período de su pensamiento. Como dijimos, en esta tarea acompañaremos la filosofía de la técnica de Simondon.

Heidegger indica que no existe algo así como un útil aislado; todo útil obtiene su sentido de una totalidad de útiles a la cual siempre pertenece, un conjunto pragmático dentro del cual se despliega la tarea fáctica de una existencia humana; por ejemplo, en el uso del martillo comparece el taller como totalidad funcional que hace que el martillo sea comprendido y utilizado, precisamente, como martillo. Heidegger aclara que la pertenencia de un útil a una totalidad no es captada de manera explícita. No es necesario, por ejemplo, que el contexto ocupacional del taller sea notado patentemente para habilitar al carpintero a concluir su construcción de la silla ${ }^{4}$. El triunfo de un artefacto depende en parte de su no-llamatividad, es decir, la ocupación no debe detenerse en la contemplación del útil, sino atravesarlo en dirección a la tarea que debe ser realizada. Heidegger indica que la ocupación se desarrolla dentro de una

\footnotetext{
${ }^{4}$ de hecho, la percatación explícita de todos los momentos nodales del plexo de remisiones sería un auténtico obstáculo al quehacer
} 
comprensión inexplícita del mundo, la así llamada comprensión primaria (Heidegger, 2004:§12a). El ser en respectividad constituye ontológicamente al útil, por ello no puede hablarse de un artefacto aislado de un entramado significativo. En cada útil late como trasfondo una totalidad respeccional, caracterizada por su significatividad. Hasta este punto he expuesto los que considero son los aportes fundamentales del primer Heidegger a una filosofía que se ocupe de la estructura de los objetos técnicos.

Ahora bien, en las diversas exposiciones del problema del artefacto y el mundo circundante técnico ${ }^{5}$ que Heidegger realiza en su obra de los años '20 se observa una constante: los útiles y medios técnicos analizados pertenecen a un estrato de baja complejidad; los ejemplos casi siempre se refieren a herramientas manuales de sencilla construcción y operación, como tizas, martillos cátedras y ventanas. En los casos en que se mencionan entes técnicos de mayor complejidad, como plantas hidroeléctricas, estaciones de tren, o canteras de extracción de minerales, las mismas no son analizadas de manera pormenorizada, los procesos y actividades que en ellas se realizan son dejados de lado por completo como si tales procesos fueran de común conocimiento. Los análisis pormenorizados del Heidegger de los '20 se dirigen a medios técnicos "hogareños". Se constatan entonces, tres elementos de la escena técnica que Heidegger toma como paradigma en el primer período de su pensamiento: 1) el modelo del objeto técnico es el artefacto manual de baja complejidad, es decir, la herramienta, o, en términos de Simondon, el elemento técnico; 2) además, el modelo del medio técnico es el taller, es decir, el medio que propicia el ensamblaje de una serie de funcionalidades y procesos sencillos (clavar, recortar, marcar, unir) y de gestos humanos simples (torcer, presionar, golpear, sostener) encarnados en entes de trabajo, con una finalidad definida y reconocible 3) Por último, el modelo del ser humano que se ubica en el centro de este simplificado medio técnico es el del artesano, es decir, el sujeto que tiene conocimiento (aunque sea antepredicativo y habitual) y control de todas las fases de la producción, y su fuerza motivadora, proyectiva y comprensora (en tanto Worumwillen) se extiende a todos los pasos de la tarea, desde la obtención de la materia prima, hasta el acabado final del producto (poco y nada se dice acerca de

\footnotetext{
${ }^{5}$ Nos parece oportuno aclarar el sentido de mundo que nos ocuparemos de revisar, dado que este término posee numerosas sutilezas a lo largo de la obra de Heidegger: nos referiremos al mundo en tanto mundo en torno técnico; consideramos que la aclaración es pertinente ya que en la obra Prolegómenos para una historia del concepto de tiempo, se establece la distinción entre dos modalidades del mundo circundante: el "mundo del trabajo" [Werkwelt] y el "mundo de la naturaleza" [Welt der Natur]. Adicionalmente, en Ser y Tiempo, se ofrece una exposición de cuatro sentidos de mundo, dos ontológicos y dos ónticos. (Heidegger, 2006, 92 y ss.)
} 
su comercialización y distribución). El productor en tanto artesano, además, no se relaciona con otros productores ni otros sistemas, no terceriza tareas y no parece depender de subsistemas que posibilitan su acción. Herramienta, taller, artesano, finalidad, función, control, intención: esta serie de conceptos fundamentales empleados a la hora de explicar la realidad técnica nos llevan a confirmar una situación reconocida por muy diversos autores: el modelo de la técnica tomado en cuenta por el primer Heidegger es la técnica artesanal. En este modelo de producción, un operario interviene directamente sobre una materia prima, y, delineando de manera clara y comprensible el proyecto a realizar, se ocupa de producir la obra que se propone, una obra para la cual posee un plan intuitivo o manifiesto. Este modelo responde, últimamente a la antigua concepción que comprende la labor técnica como impresión de una materia sobre una forma, un modelo fuertemente criticado por Simondon, como veremos más adelante.

El inconveniente de la circunscripción del cuestionamiento de la técnica al área de la técnica artesanal radica en la serie de fenómenos que quedan sin tematizar en el análisis. Estas omisiones se vuelven visibles si se estudia la realidad técnica desde una perspectiva más contemporánea como la de Simondon: Heidegger no piensa la máquina ni el entramado pluri-sistemático de tareas, instituciones y entes que Simondon denomina conjunto técnico; tampoco se considera, por ejemplo, que el medio técnico puede ser él mismo el único capaz de propiciar el funcionamiento de los objetos técnicos ${ }^{6}$. Consideramos que, de haber tenido en cuenta cuestiones de este tipo, Heidegger hubiese llegado a un esquema donde el papel central del Dasein en el mundo del trabajo se vería comprometido, y donde, en varias oportunidades, la existencia humana debería conformarse con una actitud pasiva y adaptativa ante las exigencias de la materia, de la máquina, y de los procesos naturales.

De aquí en más nos ocuparemos de analizar algunos aspectos no tematizados por Heidegger, tal como fueran destacados por Simondon, e intentaremos mostrar cómo la consideración de cada uno de estos aspectos llevaría a un descentramiento del Dasein como núcleo configurador del mundo del trabajo.

\footnotetext{
${ }^{6}$ Simondon llama medio asociado al contexto técnico en tanto espacio de ensamblaje de causalidades recurrentes. El medio asociado cumple la función de aproximar cadenas operacionales previamente no relacionadas, de tal manera que la operación de una máquina o herramienta facilite o motive los procesos de otra.(SImondon, 2007:78)
} 


\section{Primera consideración: El problema de la máquina como portadora de herramientas}

Tal vez la característica más enigmática del pensamiento técnico de Heidegger sea la falta de investigación de una temática fundamental: las máquinas. En efecto, no encontramos en ninguno de los dos períodos del pensamiento de Heidegger ${ }^{7}$ un análisis minucioso de aquellos entes técnicos -más complejos que las herramientas- que condensan y unifican variados procesos, gestos $\mathrm{y}$ movimientos.

Ya que Heidegger no tematiza esta cuestión, recurramos a Simondon para preguntar ¿Qué es una máquina? El término utilizado por el autor francés para referirse a este tipo de objeto técnico es individuo técnico. La máquina es presentada por Simondon como un ente que unifica en sí una serie de funciones, procesos y gestos humanos, y les otorga una cierta secuencialidad iterativa (Simondon, 2007:162). La máquina busca, además de la unificación de gestos (encarnados en elementos técnicos) la generación de una creciente sinergia de procesos y funcionamientos, donde cada una de las piezas (y en ellas, cada uno de los movimientos individuales) redunde en un mejor funcionamiento de las demás cadenas causales. Una máquina es una pluralidad de herramientas cuyas causalidades recurrentes han sido integradas gracias a un medio asociado. Una máquina ideal sería altamente autosuficiente, en el sentido de que cada proceso no se desarrollaría en contraposición con los demás, ni tampoco como actividad accesoria de limitación de un fenómeno producido por el funcionamiento total ${ }^{8}$, sino que cada proceso sería retomado y utilizado por otra sección de la máquina para propiciar un mejor funcionamiento de la misma ${ }^{9}$ En una máquina con un elevado nivel de sinergia de partes (en el lenguaje de Simondon, una máquina altamente individualizada) no habría callejones sin salida, todo movimiento redundaría en el mejor funcionamiento total ${ }^{10}$

En lo que a nuestra investigación respecta, observamos una destacable característica de la máquina, que, debido a la omisión de la temática, y la focalización en el objeto técnico en tanto elemento, y no como individuo, no aparece en los estudios heideggerianos: la máquina también es portadora de

\footnotetext{
${ }^{7}$ Si damos por válida esta distinción entre dos períodos de su pensamiento.

${ }^{8}$ Como por ejemplo, una serie de difusores de calor añadidos externamente a una máquina que provoca elevadas temperaturas de funcionamiento.

${ }_{9}^{9}$ Por ejemplo, en una máquina donde la elevada temperatura disminuye la viscosidad e incrementa la fluidez y circulación del líquido que se usa para refrigerarla.

${ }^{10}$ Desde luego, esta creciente autosuficiencia no significa que se puedan suspender fenómenos que tienden a cierta inevitable ineficiencia del aparato, como el fenómeno físico de la pérdida de calor en todo intercambio térmico.
} 
herramientas (Simondon, 2007:37) Esto la coloca en un lugar mucho más cercano a la realidad humana. En el esquema de Heidegger sólo el Dasein era capaz de construir, portar y utilizar herramientas. Simondon, en contraste, nos indica que en el esquema artesanal el ser humano funcionaba él mismo como medio asociado, es decir, él cumplía la función de fusionar las herramientas en un individuo técnico. El artesano era el agrupador y aglutinante de las herramientas. Desde luego el núcleo de este papel aglutinante era la finalidad del quehacer técnico. Tal vez, conjetura Simondon, de este carácter unificador provenga la tradicional consideración del trabajo artesanal como "más noble" que el trabajo industrial (Simondon, 2007:97). Simondon nos indica que antes del advenimiento de las técnicas industriales, el ser humano era el soporte de la individuación técnica, pero, en nuestros días, el soporte es la máquina. El ser humano ya no tiene la prioridad en la portación de herramientas (Simondon, 2007: 98).

A partir de la revolución industrial, numerosos gestos y actividades que aparecían condensados en herramientas se reunieron en objetos técnicos individuales que, movilizados por una fuente de energía como el vapor, llevaron a una repetición iterativa de los gestos que antes eran desarrollados por el artesano o el grupo de artesanos. Vemos como a partir de la llegada de la máquina, el papel central del Dasein como portador de herramientas queda comprometido. La máquina se acerca y asemeja al ser humano. De hecho, debido a que la proximidad es tan acentuada, una máquina puede llegar a reemplazar a un operario humano, lo mismo no puede afirmarse de los elementos técnicos (herramientas): un martillo no puede reemplazar a un obrero, pero sí una prensa hidráulica automatizada. "si el hombre siente una frustración ante la máquina es porque ella lo reemplaza funcionalmente en tanto que individuo" (Simondon, 2007:98). El ser humano es uno más entre las máquinas, y no un dominador.

Sin embargo, el ser humano sigue hoy buscando un lugar estable junto a la máquina, pero en los términos tradicionales de dominación; él quiere seguir siendo individuo técnico y centro unificador, pero ha sido desplazado de esta función hace tiempo. Este estado de "falta de morada" genera malestar que puede desembocar en la alienación del obrero en la escena técnica contemporánea. En el SXVII el ser humano seguía siendo un individuo técnico, centro de herramientas. En el SXIX, hacen su aparición sinnúmero de individuos técnicos completos, pero estos sólo reemplazaban el trabajo de animales (la máquina de vapor, al principio, sólo reemplaza al caballo para llevar vagones), por ello no generaban frustración en la experiencia humana. la frustración comienza cuando la máquina se instala en el lugar en el que el ser humano actuaba como ensamble de herramientas y procesos, allí es donde se ve 
reemplazado y desplazado. En efecto, las máquinas que el obrero destruye en las revueltas de trabajadores son las que portan herramientas, como por ejemplo, el revolucionario telar mecanizado (Simondon, 2007:132)

A este primer factor que asemeja la realidad del ser humano a la de los individuos técnicos se le suma un segundo aspecto que acrecienta el distanciamiento del Dasein del centro de la escena técnica:

\section{Segunda consideración: la esencia del objeto técnico no es la función}

Según la exposición de Simondon, la esencia del individuo técnico (máquina) no está en su función, sino en su genealogía y la sinergia de operaciones y procesos. Por su parte, Lo característico de un elemento técnico (herramienta) es encarnar un gesto humano, como el presionar, golpear o torcer.

Analicemos cada una de estas afirmaciones: un gesto no es lo mismo que una función. Un gesto es un movimiento (usualmente manual) circunscripto, que puede ser repetido muchas veces. Un gesto puede ser encarnado o facilitado por un sinnúmero de herramientas, el gesto, en sí mismo, no tiene finalidad unívoca (Simondon, 2007:97). El acto de torcer puede aplicarse a la función de perforar un corcho, de provocar el desplazamiento horizontal o ascendente de materiales, o de quebrar las cervicales de un condenado a muerte. Los gestos constituyen una instancia anterior a las finalidades. A diferencia de la función, el gesto no se haya aferrado a la realización de alguna tarea. La direccionalidad y aplicación de los gestos son las que darán origen a las "familias" de $\operatorname{artefactos}{ }^{11}$. Esta perspectiva contempla una importante cuestión adicional: el fenómeno del rediseño, es decir, la plasticidad de un artefacto para ser aplicado a una función diferente de aquella a la que más frecuentemente se aplica el gesto. Mientras el objeto esté abierto al gesto, podrá ser empleado en la dirección que se necesite. Un zapato puede martillar porque puede encarnar el acto de golpear, pero no una soga $o$ un alfiler.

Con todo, la temática en la que la filosofía de la técnica de Simondon muestra su máxima expansión y profundidad no es en el análisis de las herramientas, sino en el de las máquinas. Este tratamiento es aún más contrastante con Heidegger. De hecho, este es el punto que podría provocar una verdadera torsión en la interpretación del papel del ser humano y de la estructura del mundo del trabajo.

\footnotetext{
${ }^{11}$ no sólo hay martillos para clavar, hay martillos para alisar, para moler, forjar, estimular reflejos nerviosos; hay martillos para madera, para acero, y para hueso; incluso podemos descubrir que el martillo (en una versión modificada) también se encuentra integrado en máquinas como el piano y en la máquina de escribir
} 
De la falta de tematización del problema de las máquinas en el sistema heideggeriano podría inferirse que la estructura de las mismas se pliega al esquema de las herramientas. Sin embargo, ambos tipos de entes técnicos exhiben notorias diferencias.

Según Simondon, lo más relevante en la determinación del modo de existencia de un individuo técnico (y aún en un sentido bastante vago y genérico) es la génesis y creciente individuación de dicho objeto, es decir, el proceso por el cual cada una de sus partes tiende a una mayor sinergia de funcionamientos, o sea, el establecimiento de una cadena de causalidades recurrentes que desemboca en el mejor funcionamiento del todo. La esencia del artefacto complejo, indica el autor francés, está en su génesis, el individuo técnico va creciendo en definición (evoluciona) a medida que aumenta su complejidad y acoplamiento armónico de partes y movimientos. Lo propio del individuo técnico es provenir de una estirpe de variaciones con creciente interdependencia de elementos. Los útiles artesanales son ampliamente vagos y genéricos (abstractos, en la terminología de Simondon), los artefactos industriales han aumentado su individuación, de cualquier manera, la tecnicidad no tiene que ver con la reducción y focalización de sus posibilidades de aplicación, sino con la retroalimentación de las partes ${ }^{12}$. La evolución de un objeto técnico, y por tanto, su problematicidad ontológica se hace más manifiesta a medida que ellos se acercan al nivel de máquinas. De hecho, parece percibirse en la obra de Simondon un cierto "evolucionismo" de las máquinas, donde su proceso de individuación ocurriría con independencia de las funciones que los productores y usuarios quisieran darles. Dentro de esta perspectiva, la función del "gen" la cumpliría el elemento técnico, depositario de la tecnicidad, que es lo que efectivamente se transmite de un individuo a otro y puede dar origen a una nueva estirpe.

Volvamos al tema central de esta investigación: ¿cómo repercute la perspectiva que nos indica que la esencia del objeto técnico (ya sea en el nivel de las máquinas o de las herramientas) no está dada por su función, sino, respectivamente, por el gesto impreso, o por la sinergia de procesos y causalidades recurrentes, en la imagen heideggeriana de la centralidad del Dasein en la escena técnica? El punto en el que la deflación del carácter

\footnotetext{
${ }^{12}$ El motor de combustión interna ha llegado a su forma actual (altamente individualizada) como resultado de una historia de versiones y ensayos, donde se fueron superando modelos más rudimentarios, que simplemente adherían partes independientes a una estructura o chasis integrador que forzaba la unión de las causalidades, pero con una elevada pérdida de energía, e ineficiencia de procesos, o con un alto número de efectos accesorios e innecesarios para el funcionamiento del individuo.
} 
definitorio de la determinación humana de la direccionalidad de la escena técnica y sus objetos se vuelve más manifiesta es, sin dudas, en el nivel de las máquinas. La individuación y concretización de los objetos técnicos se realiza con independencia de las funciones particulares que los productores y usuarios quieran darles.

Dado que la génesis de los objetos técnicos, es decir, el carácter central de su estructura, se aprecia mucho mejor en un aparato muy concretizado y de alta coherencia interna, será precisamente la investigación de las máquinas y no la de las herramientas (modelo estudiado por Heidegger) la más capaz de revelarnos el modo de existencia de estos objetos, es decir, su tecnicidad. El fenómeno de la funcionalidad sigue cumpliendo un papel relevante en la elucidación de la estructura ontológica, pero, a diferencia del estudio de los útiles artesanales, en los individuos técnicos el "para qué" se encuentra más en las piezas analizadas por separado que en el todo funcional. El "para qué" pasa a ser interno a la máquina, así, una pieza justifica su participación en el todo concreto en una determinada finalidad asociada a la operación de este todo: "para que no se recaliente", "para que no se sacuda", "para limitar emanaciones de gas". Si recordamos que las piezas de una máquina suelen ser elementos técnicos y no individuos complejos observaremos que el problema de la funcionalidad es mucho más central para la definición de los útiles artesanales que para la de las máquinas.

Al no dedicarse intensivamente al estudio de las máquinas (artefactos complejos, surgidos de un universo industrial) Heidegger no llega a explorar la cuestión de la tecnicidad de los artefactos precisamente en el punto en que ella se vuelve problemática, es decir, en el punto en el que el útil se independiza del usuario, incluso en su constitución esencial ${ }^{13}$.

Las aplicaciones de un individuo técnico no están necesariamente predefinidas en su construcción; una misma máquina (en tanto conjunción sinérgica de partes) puede ser utilizada para finalidades diversas ${ }^{14}$. La consideración de la máquina como modelo - y no la herramienta- nos muestra un ente técnico en cierto modo desarraigado de los intereses concretos humanos; esto conlleva el descentramiento del papel del usuario y productor. La propuesta de Simondon es pensar la máquina desde ella misma. La colocación de la

\footnotetext{
${ }^{13}$ Desde luego, reconocemos que la intención de los escritos de Ser y Tiempo no era la elucidación de cuestiones asociadas a la tecnicidad de los artefactos.

${ }^{14}$ Un motor de combustión interna puede aplicarse al movimiento de un automóvil, o de una bomba de agua. Una cámara de niebla no es otra cosa que un nebulizador consagrado a las tareas de la ciencia, con un par de compartimentos agregados. Una vieja imprenta manual de dos rodillos es esencialmente la misma máquina que se usa para cortar masa y fabricar distintos tipos de pasta.
} 
máquina como centro de la investigación nos muestra que ella es el tipo de ente que más tiene para decir acerca de la ontología de los objetos técnicos. Esto se debe a que ella evoluciona, ella es un artefacto viviente, en permanente mutación como resultado de un juego de fuerzas internas; las herramientas, en cambio, son unidimensionales en su estructura, por ello, al analizarlas, el papel rector y semantizante del ser humano aparece resaltado. Prueba de esto es que la forma de los martillos casi no ha variado a lo largo de miles de años, dado que el gesto que ellos concretizan no se modifica. Los útiles artesanales prácticamente no tienen evolución, por ello su explicación necesariamente ha de aterrizar en la figura d el usuario que es lo que en efecto varía y evoluciona.

\section{Tercera consideración: el objeto técnico como configurador de espacio}

Según la filosofía del primer Heidegger, la praxis cotidiana se relaciona inicialmente con las áreas de influencia y de acción de los aparatos con los que trabaja, cada artefacto se instala en un "lugar propio", esto es, una ubicación significativa (no inicialmente geométrica o topológica) en el contexto del mundo circundante desde donde puede relacionarse con los demás objetos técnicos del entramado remisional. La interrelación de los lugares propios de cada artefacto da como resultado una "zona", esto es, un ámbito significativo de ubicaciones relativas a la praxis. De este modo, la experiencia originaria del espacio aparece en la misma interacción del trato técnico en el mundo; así, el "arriba" aparecerá como "en el estante superior" o como "demasiado alto"; el "abajo" como "en el cajón inferior", o en el "hay que agacharse". Esta intuición es muy relevante para la filosofía de la técnica, puesto que le otorga al artefacto la capacidad de modelar y abrir formas del espacio que se emparentan con sus exigencias funcionales. El tratamiento de Heidegger abre una posibilidad de investigación que él mismo no explora: el carácter determinante de la estructura material y funcional de un artefacto a la hora de definir su posición en el espacio técnico ${ }^{15}$. Un estudio de estas características terminaría por mostrar a un Dasein desplazado a la hora de establecer el sentido del mundo técnico.

Vemos que Heidegger reconoce una forma originaria de la espacialidad que se asocia íntimamente con el quehacer productivo. Esta modalidad pre-teórica pone en libertad un ordenamiento de zonas de carácter significativo. Ahora bien, en el planteo de Heidegger, la configuración espacial-semántica del mundo responde indefectiblemente al proyecto fáctico que en cada caso despliega una

${ }^{15}$ En este sentido, la cama "debe" colocarse directamente debajo del ventilador de techo. 
existencia humana. Esto significa que el núcleo de ordenación espacial es siempre un último "para-qué" que siempre nombra a un "paraquién"(Worumwillen). La confirmación de esta centralidad la encontramos en dos actitudes fundamentales por medio de las cuales el ser humano se despliega en el mundo: la actitud desalejante (Heidegger, 2006:131) y la ordenación espaciante (Heidegger, 2006:136) El centro de la configuración espacial siempre es el Dasein que proyecta.

Ahora bien, ¿qué pasaría si las herramientas, máquinas y procesos productivos trajesen aparejadas una serie de "sugerencias" zonales? ¿No ocurre acaso que los entes técnicos "buscan" ser localizados en ciertas posiciones y ubicaciones, y rechazan otras, so pena de no funcionar de manera adecuada, o peor aún, de interferir de manera destructiva con los procesos de otros entes técnicos? El diseño (a cargo del productor humano) del artefacto sólo a veces predetermina la ubicación futura del mismo. En la mayor parte de las oportunidades, los procesos funcionales de un ente técnico son solidarios con los de otro, pero generan interferencias con los de un tercero, y es esta interrelación de procesos y funciones la que determina, con independencia de las decisiones fácticas del productor, el lugar que ocupará el artefacto en el espacio significativo. Las chimeneas en el siglo XVI, las radios de los años '20, y los televisores de los años ' 60 , todos producían un cierto "efecto centrípeto" que llevaba (y sigue llevando) a que los muebles de descanso se ubiquen delante de ellos, y favorezcan la reunión. Sin embargo, este papel "atractor" de útiles de descanso no estaba inscripto en el diseño de dichos artefactos, sino que se hizo presente en el despliegue cultural de sus potencialidades "socializantes". Un acondicionador de aire (en modo calefactor) que se ubica en lo alto de una habitación de doble altura no logrará aumentar la temperatura de toda la habitación, puesto que el aire caliente tiende a quedarse en la parte elevada; aún más dificultoso será el proceso de calentamiento si el aparato se ubica junto a grandes ventanas de vidrio en época invernal; en este caso vemos como la fractura funcional no se encuentra en la construcción o avería del aparato, sino en su posición en el espacio y en su cercanía con otros entes técnicos. Los entes técnicos sugieren el espacio en el que deben ser colocados. Esta exigencia de localización es mucho más visible cuando los procesos físicos que acontecen en el objeto técnico acarrean peligros para la vida humana, o podrían dar inicio a cadenas causales destructivas si acaso su localización se superpusiese con la de otro sistema artefactual (pensemos, por ejemplo, en una central eléctrica construida en las proximidades de una gasolinera)

La instalación de un aparato en una determinada ubicación no surge solamente del proyecto del ingeniero u operario, sino muy a menudo de las 
relaciones funcionales que una máquina tiene en referencia a otras y al ambiente en el que opera; por ejemplo, un estudio de grabación deberá tener en cuenta la interferencia que los micrófonos y amplificadores pueden generar entre sí, al tiempo que aísla físicamente ciertos instrumentos y la misma consola de mezcla; algo similar ocurre en la exigencia de limpieza y temperatura constante en espacio técnico de un laboratorio de agentes patógenos o una fábrica de microprocesadores.

Vemos cómo las máquinas y sus redes de interconexión llevan a modificar el ambiente en el que se instalan. La misma arquitectura del lugar de trabajo debe considerar estos aparatos, su peligrosidad o la necesidad de su cercanía. La disposición del espacio técnico responde sólo transversalmente a la intención del agente técnico. Una vez más, se observa que la máquina y el ser humano se encuentran en una posición parcialmente análoga (puesto que ambos son portadores de herramientas, e influyen en la configuración del espacio). Las máquinas son capaces de diseñar su propio ambiente y de reconfigurar el espacio, y lo hacen por medio de la exigencia fáctica de fenómenos, procesos y materiales.

Analicemos un caso adicional, en un intento por acrecentar la relativización de la centralidad del Dasein en la configuración de sentido del mundo del trabajo: nos preguntamos ¿dónde se construyen los talleres (modelo heideggeriano del espacio técnico)? ¿Acaso en el interior de las casas, en medio de las alacenas, los sillones, las bibliotecas y las cocinas, o más bien en un espacio separado, y expresamente alejado del terreno en el que ocurre el descanso, la alimentación, y la higienización?. ¿Es por mero capricho que, casi en la totalidad de los casos, las camas son expulsadas de los talleres? ¿O esta práctica responde a un ordenamiento inherente a la misma estructura de un taller?. Resulta claro que el espacio técnico del taller trae consigo una serie de factores que resultan incompatibles con la actividad del descanso, aspectos que incluso podrían poner en riesgo la salud de quien buscase permanecer en su interior más tiempo que el necesario para completar los proyectos técnicos. Los talleres traen consigo ruidos, olores, desperdicios o niveles de temperatura que resultan aceptables para el que trabaja, pero inaceptables para el que descasa.

La instalación de las camas lejos de los talleres no es antojadiza. En el diseño del espacio del trabajo y el espacio del descanso están necesariamente previstas o tenidas en cuenta las características inherentes a cada actividad y las causalidades y procesos que en ellas se desarrollan. La cadena causal del acto de soldar dos metales necesariamente se encontraría en interferencia con la cadena causal del acto de dormir. El diseño y disposición del espacio técnico tiene en cuenta, aunque no se la detecte de manera expresa, una amplia y compleja serie 
de procedimientos, funcionamientos, actividades y causalidades que no son precisamente centrales al proceso de producción, pero, incluso en su carácter accesorio o concomitante, son suficientes para exigir un rediseño del espacio, en función de su manera de afectar el vivir y trabajar humanos. Ante esos factores, la actividad humana que ordena áreas significativas no puede más que adaptarse, o replantear la distribución del mundo circundante en función de una o varias causalidades intersecantes que provocarían un choque (incómodo en el mejor de los casos, catastrófico en el peor) de vectores pragmáticos incompatibles.

El espacio del trabajo y el del descanso no suelen convivir, y esto no ocurre únicamente por una serie de convenciones sociales e históricas, sino -y acaso principalmente- porque las operaciones que se realizan sobre la materia en el acto de producción vehiculizan o desencadenan un entramado de procesos $\mathrm{y}$ consecuencias que llevan a las dos tareas a distanciar sus encadenamientos, y en esa misma separación, a dividir físicamente las áreas en las que cada una de ellas será llevada a cabo.

Lo que aquí se intenta afirmar es que el mundo circundante expuesto y analizado por Heidegger en Ser y Tiempo, puesto que el mismo queda asociado al modelo artesanal que no considera el problema de los conjuntos técnicos, resulta insuficiente para dar cuenta de la complejidad de la realidad técnica contemporánea en términos espaciales y de interrelación pragmática de sistemas y causalidades ${ }^{16}$. Los procesos, los artefactos, y los subsistemas también son capaces (y tal vez lo sean en primera medida) de definir los límites del Werkwelt. El mundo circundante no se abre solo en función del interés del Dasein, también se abre hasta donde lo permiten o lo exigen los materiales, las máquinas, las causalidades técnicas y las consecuencias implicadas en el despliegue de todas ellas.

En numerosas oportunidades el rol del Dasein es pasivo ante lo irrefrenable de un fenómeno físico que ocurre en el contexto de un medio técnico. Por ejemplo, un resonador magnético -si se desea evitar las interferencias con el equipamiento de medición- deberá ser instalado en el interior de una jaula de Faraday; una máquina de rayos $\mathrm{X}$-si se desea evitar el riesgo a la salud de los usuarios- deberá ser construida teniendo en cuenta algún tipo de sistema aislante, por ejemplo, paredes de plomo; un (ya antiguo) taller de revelado fotográfico sólo puede ser construido en un ambiente oscuro, o de muy baja luminosidad para proteger el material fotosensible; una fábrica de microprocesadores no

\footnotetext{
${ }^{16}$ Desde luego, reconocemos que este no era el objetivo de los escritos de los años '20, pero sí es el intento de la presente investigación, a saber, extraer los elementos principales de una filosofía heideggeriana de los entes técnicos para luego amplificarla y actualizarla por medio de la perspectiva Simondoneana de una escena técnica expandida.
} 
tolerará el ingreso de polvo al espacio técnico, por lo cual la misma deberá tomar numerosas medidas refrigeración y filtrado de aire, las cuales modificarán radicalmente la estructura y diseño (interno y externo) de la fábrica, y también su modo de relacionarse con los demás subsistemas que están implicados en las tareas de construcción.

Todas estas situaciones definen, con independencia de la conveniencia del Dasein, la forma, figura, tamaño, y localización del espacio técnico. Casi todos los espacios técnicos adquieren su configuración fáctica no porque el ser humano lo prefiera de tal o cual modo, sino porque de otra manera, los procesos físicos buscados no ocurrirían -o interferirían unos con otros- y la actividad técnica se detendría.

Del breve análisis realizado puede extraerse la siguiente conclusión: la ordenación espaciante es prerrogativa del Dasein, puesto que él es el único ente que vive en el modo de la des-alejación y la configuración de zonas. Sin embargo, en numerosas oportunidades, el ser humano sólo puede adoptar una actitud pasiva o adaptativa ante las exigencias de ubicación que los objetos técnicos aportan a la experiencia espacial del mundo.

\section{Cuarta consideración: Los límites de la materia}

En el punto anterior resaltamos el modo en que las estructuras internas e interconexiones procesuales de los objetos técnicos son capaces de torcer la ordenación del espacio del trabajo en una dirección u otra. Ahora bien, existe un factor fundamental que también debería ingresar en la consideración del corrimiento de la existencia humana del centro de la escena técnica: las causalidades y procesos naturales que operan en el artefacto y que posibilitan, determinan y limitan su funcionamiento. La atención a estos factores causales llevarán, por otra vía, a la disolución de los límites del mundo circundante artesanal.

En la relación pragmática cotidiana con el mundo tecnificado, el usuario tiene una experiencia precientífica de fenómenos y regularidades que operan de antemano en la relación entre los objetos. El operario puede obtener, por ejemplo, una experiencia del caer de una silla, de una piedra, de un florero o de un edificio. Esta experiencia directa y pre-teórica de las uniformidades de procesos del mundo puede entenderse como una comprensión de habitualidad del "estilo global empírico" o "estilo causal universal" (Husserl, 2008:73) del mundo circundante. El hacedor de espadas comprende que el acero cambia su color a medida que se lo calienta, aunque desconoce la ley de Rayleigh-Jeans, que 
predecía, hasta principios del siglo XX, la emisión de radiación térmica de los cuerpos. También comprende que ciertos colores (asociados a temperaturas) son los indicados para forjar y otros son los indicados para templar el metal. El herrero también sabe que si se intenta templar el metal (sumergiéndolo en agua, por ejemplo) cuando el acero brilla en un tono inadecuado, el proceso podría finalizar en la ruptura de la espada. Resulta manifiesto que el operario comprende que ciertos procesos y encadenamientos fenoménicos duermen en la materia sobre la que él trabaja, y, para que su tarea sea exitosa debe adaptarse a dichos encadenamientos causales.

Lo que intentamos afirmar es que la actividad humana ya se encontraba delimitada por procesos inherentes a la materia, mucho antes de que estos fueran tematizados; en otras palabras, la comprensión primaria del quehacer productivo acarrea una comprensión primaria de los límites que los procesos latentes traen a ese quehacer. Estos límites le revelan al operario que el centro de la escena técnica no está ocupado por él, sino que su posición está nivelada con la de la materia y los artefactos. Es en este sentido que Simondon afirma "el mejor fontanero no puede hacer que el agua suba por encima de los 10 metros 33 centímetros" (Simondon, 2007:221)

De hecho, la comprensión tematizante de las regularidades físicas ni siquiera es necesaria para la construcción de artefactos que hagan uso de dichos principios, (baste con recordar que la máquina de vapor para uso industrial fue inventada más de 50 años antes que la formulación de las leyes que explican su funcionamiento). En un proceso posterior, y derivado, las regularidades del quehacer involucrado con la materia pueden ser formuladas en forma de leyes científicas que, incluso luego de su derivación continuarán delimitando las actividades productivas humanas.

El resultado de la toma en consideración de los límites causales existentes en todo proceso técnico nos muestra, una vez más, que la descripción del ser humano en tanto punto de originación de sentido y organizador semántico del mundo del trabajo resulta insuficiente para dar cuenta de la los procesos contemporáneos de explotación de materia prima y de producción de objetos técnicos. La finalidad, arrojada por el agente humano, indefectiblemente colisionará con el ordenamiento previo de los vectores causales que duermen en el interior de todo proceso técnico. Por ejemplo, no cualquier tipo de uranio, sino sólo el isótopo 235 puede ser utilizado para sostener la reacción en cadena que posibilita el funcionamiento de una central de energía nuclear. Una vez más, si se actualiza la perspectiva, y se suspende el modelo del taller y del artesano, observaremos un corrimiento de la relevancia del ser humano en la escena técnica, este desplazamiento conduce a una equiparación o nivelación de la 
relevancia organizativa del productor con la de las máquinas, al mismo tiempo que se observa que su intención productiva no es suficiente para explicar la complejidad del mundo del trabajo.

Ahora bien, como explicamos al comienzo, no intentamos afirmar aquí que los artefactos y sistemas técnicos tengan mundo o acceso al fenómeno existencial del sentido, pero sí afirmamos que su modo de venir a la presencia trae una serie de exigencias ante las cuales el Dasein (punto donde acontece el sentido y la comprensión) sólo puede comportarse de manera adaptativa, reestructurando el mundo circundante de modo que ellas sean tomadas en consideración, de lo contrario, el Werkwelt se vendría abajo por entrecruzamientos de causalidades y procesos incompatibles. Será suficiente pensar en los trabajadores muertos o heridos durante la construcción del puente de Brooklyn: su trabajo de excavación se realizaba en enormes cajas de madera sumergidas (caissons) en el lecho del Río Este, sin tomar en consideración los problemas que los repentinos cambios de presurización, o peor aún, el uso de explosivos dentro de ambientes presurizados causaban en los operarios. Un Werkwelt que no considera las causalidades físicas acaba por colapsar, a veces, llevándose consigo al ser humano que trabaja en él. Desde luego que sólo el Dasein configura mundo del trabajo, pero no puede hacerlo sin atender a las exigencias físicas, logísticas, históricas y sociales que las máquinas y los subsistemas le presentan de manera insistente. Una perspectiva analítica del mundo circundante que considere estos aspectos, necesariamente acabará por desmantelar los límites hogareños y artesanales del taller imaginado por Heidegger, y llevaría a una más precisa expansión del mundo circundante en un conjunto técnico.

\section{Quinta consideración: la insuficiencia del modelo hylemórfico}

Es manifiesto que el problema de la técnica ha sido una cuestión de gran relevancia en la filosofía de Heidegger desde sus comienzos. Las lecciones de Friburgo se ven marcadas por un estudio de la filosofía aristotélica y una recuperación de la téchne como un modo del desocultamiento. Las obras de los años '20 (que culminan en la analítica existencial) toman al útil como ente paradigmático del trato cotidiano del Dasein en el mundo, y desde esta vía de acceso, se ocupan de elucidar las estructuras ontológicas que definen a la existencia humana. Las obras de los años ' 30 y posteriores están signadas por una aproximación crítica al fenómeno de la técnica, donde ella se convierte en el último eslabón en la historia del olvido del ser, y el punto culminante de la metafísica occidental, dominada por el principio de razón suficiente. 
Heidegger indica que la concepción aristotélica de sustancia (principio que se volverá central para la metafísica occidental) surge de una interpretación griega del mundo que ponía el centro en el ente producido ${ }^{17}$, así, todo lo que llega a ser, lo hace por aplicación de un éidos (como figura del producto terminado en la mente del productor) que se convierte en la forma del compuesto sustancial. Producir algo es aplicar una forma a una materia. Distinto es el caso de los entes que no requieren de producción, los physei ónta, y de los entes eternos, aei ónta. (Heidegger, 2000:150)

Si bien Heidegger logra superar este modelo a la hora de tematizar la existencia humana y el sentido del ser en general, el esquema sigue rigiendo en el caso particular del análisis de la estructura de los objetos técnicos y de la relación humana con ellos. El estudio de entes técnicos por medio de un modelo que reserva un lugar central para la función (lo que hemos llamado intencionalismo) es heredero de la concepción que prioriza el modelo de la producción en tanto aplicación de una forma a una materia.

En otras palabras, el estudio Heideggeriano de la estructura de los entes técnicos del período de Ser y Tiempo se inspira en el esquema hylemórfico. Heidegger libera el análisis de las ataduras del modelo de la producción e hylemorfismo a la hora de estudiar la existencia humana, pero no a la hora de estudiar los entes de uso. En contraste, Simondon realiza una incisiva crítica a dicho modelo como esquema explicativo de la estructura de los objetos técnicos. Él nos indica que la noción de aplicación de forma a materia representa una perspectiva simplista e ingenua, que pierde de vista la complejidad de las actividades técnicas.

Según Simondon, si se analiza un proceso productivo simple, se hará visible la insuficiencia del modelo materia-forma. Por ejemplo, para que pueda ser producido un ladrillo de barro, existe una serie de fases previas y condicionantes; si estas fases no son predispuestas, el amoldamiento del ladrillo jamás ocurriría. No cualquier barro se adapta a cualquier molde. La materia debe ser limpiada y acondicionada buscando evitar burbujas y grumos; a su vez, si el barro se encuentra en un estado demasiado líquido, el mismo se escaparía por las mínimas hendijas del molde de madera. Esto significa que la "materia", es siempre ya una materia preparada, manufacturada, intervenida, preindustrializada. En la realidad técnica contemporánea casi no existe contacto con materia prima ${ }^{18}$ "a secas".

\footnotetext{
${ }^{17}$ De aquí proviene la interpretación de ousía como hacienda, enseres, posesiones, objetos de uso (Heidegger, 2002:58), y la concepción del sentido originario griego del ser como ser-producido. (Heidegger, 2002:57)

${ }^{18}$ Entendemos aquí "materia prima" en un sentido no aristotélico, sino de diseño industrial y
} 
En efecto, Simondon indica que quien verdaderamente aplica la forma a la materia no es el productor, sino el molde (un objeto técnico). El fenómeno de "aplicación de forma a materia" queda por fuera de la experiencia humana, ya que ocurre en la intimidad de la relación entre el molde y el material. El productor simplemente aproxima las causalidades ya operantes para que ocurra una solidaridad de una con otra, pero no tiene un papel rector en el ensamblaje de las mismas. Esto se confirma en el hecho de que el material debe ser preparado de antemano para facilitar la solidaridad funcional con el molde. Para que la aplicación de una "forma a una materia" ocurra de hecho, hace falta un conjunto técnico que fusione zonas operativas. En otras palabras, la condición de la adquisición de la forma es el sistema y el conjunto técnico, esto es, la arcilla y el molde, preparados el uno para el otro, dentro de un contexto de zonas funcionales ensambladas.

La herramienta no está hecha solo de materia y forma, está hecha de elementos técnicos elaborados de acuerdo con ciertos esquemas de funcionamiento y reunidos en una estructura estable por la operación de fabricación. La herramienta recibe en ella el funcionamiento de un conjunto técnico. Para hacer una buena hachuela, es preciso el conjunto técnico de la fundición, de la forja. (Simondon, 2007:92)

La forma (si es que aún se puede emplear esta terminología) no se deja aplicar simplemente a la materia; la materia "decide" qué es lo que recibe y lo que rechaza. Es por ello que para que una figura o forma sea aplicada a un material se vuelve necesario un acondicionamiento de la materia y del medio técnico para facilitar la impresión. Simondon indica que materia y forma son en verdad dos semicadenas técnicas (Simondon, 2007:259). El agente técnico que dirige un trabajo se ocupa de la materia y de la forma, pero no de la operación misma que permite la adquisición de la forma, esto significa que en el esquema hylemórfico los términos son nítidos pero la relación oscura. Simondon añade que habría que tratar la adquisición de forma como una operación técnica particular en lugar de tratar todas las operaciones técnicas como casos particulares de la adquisición de forma, la cual, ni siquiera es conocida con claridad.

Simondon indica algo más: la preeminencia de la forma sobre la materia, y como consecuencia, la completa sumisión de la materia, proviene de la visión del amo, es decir del dueño de primitivos sistemas de producción, que jamás ha conocido lo que ocurre en el interior de un taller ${ }^{19}$. La experiencia de la

económico, como "aquello de lo que está hecho el producto".

${ }^{19} \mathrm{Si}$ este desconocimiento se refiriese a lo que ocurre en las fábricas en lugar de los talleres, la 
producción lleva descubrir que la materia tiene sus propias exigencias. Esta noción pone en crisis la idea misma de preeminencia del diseño imaginativo por sobre la materia a ser modificada. Esta posición se encuentra en la base del descentramiento de la figura humana en el mundo tecnificado. El ser humano ya no sólo domina e imprime ideas sino que se adapta a las exigencias de sus aparatos, a los materiales y a los fenómenos físicos que tienen lugar en esta compleja interrelación. Visto desde Simondon Heidegger mantendría vigente la visión del amo, del productor omnipresente, y determinante; por ello el Dasein sigue siendo el centro de su esquema del mundo técnico. Simondon, en cambio, pone prioridad en la máquina (individuo técnico) y en los conjuntos técnicos, por ello reconoce que la materia y el artefacto tienen su propia "voz" y que también ellos modelan el mundo.

\section{Conclusión}

Los estudios académicos acerca de la técnica en la filosofía de Heidegger tienden a concentrarse en el segundo período de su pensamiento. En este trabajo intentamos una mínima recuperación del primer período de su pensar y una actualización y amplificación de los conceptos allí expuestos, para volverlos más aptos para dialogar con filosofías de la técnica contemporáneas. En esta oportunidad no nos concentramos específicamente en la indicación de los aportes fundamentales del primer Heidegger a la ontología de los entes técnicos, sin embargo algunas cuestiones fueron mencionadas. Resumidamente podríamos decir -recuperando las conclusiones de estudios anteriores de nuestra autoría- que las nociones principales de la ontología de la técnica del primer Heidegger son: 1) El objeto técnico es el ente con el que la existencia humana se relaciona de manera inmediata y regular. El estudio del artefacto se engarza en un análisis de la cotidianidad media; 2) La esencia del artefacto se encuentra en su remisionalidad. Adicionalmente, en el centro de esta remisionalidad siempre encontramos a un usuario humano que direcciona la aplicación del útil en función de su propio proyecto (esta postura puede denominarse "intencionalista"); 3) El objeto técnico se instala en un mundo circundante, y obtiene de él (y del proyecto del usuario) su sentido y su relevancia; 4) El objeto técnico es capaz de modelar el espacio significativo de la cotidianidad; 5) El artefacto se caracteriza por su no-llamatividad. La atención debe atravesarlo en dirección a la tarea que se desea realizar; 6) Existen formas deficientes de la

afirmación podría aplicarse a Heidegger. 
ocupación. Si el artefacto se descompone o se ausenta, se vuelve manifiesto (aunque aún no teóricamente) el entramado remisional de los "para qué". En esta red de sentido pragmático se acusa el mundo circundante; 7) el artefacto es capaz de condensar y compartir información sobre el medio técnico; 8) La naturaleza hace su aparición (aún no teoréticamente) en la misma estructura del útil, a saber, como material del que está hecho el objeto técnico, como materia prima sobre la que este artefacto opera, o como medio que se presupone para el despliegue de la operación técnica.

El hecho de que la intención de Heidegger no haya sido la de fundar una filosofía de la técnica (en tanto reflexión filosófica acerca del modo de existencia de los objetos técnicos) no nos impide a nosotros realizar una compilación y actualización de su ontología de los artefactos. En esta línea también intentamos destacar las limitaciones de las reflexiones del autor alemán, y procuramos expandir las reflexiones por medio de un diálogo con cinco nociones estudiadas por G. Simondon: 1) el individuo técnico (máquina), el tipo de objeto de uso que predomina en la interrelación técnica actual, es portador de herramientas, esto es, está formado por un conjunto de útiles de baja complejidad que se unifican favoreciendo encadenamientos causales y sinergias de operaciones. Ya no solo el ser humano hace uso de herramientas, también los objetos técnicos emplean objetos técnicos. 2) la funcionalidad cumple un papel secundario en la determinación de la esencia del objeto técnico. El carácter fundamental para determinar la estructura de una herramienta es el gesto, y, en el caso de las máquinas, la sinergia de operaciones y su genealogía; si la función, en el esquema clásico heideggeriano, remite últimamente al Dasein, y la misma ha dejado de ser determinante, entonces el Dasein queda alejado una vez más del centro del mundo del trabajo. 3) En el objeto técnico se encarna una serie de procesos que frecuentemente establecen de antemano las interrelaciones que podrán tener con otras máquinas y herramientas. Esta interrelación potencial establece conexiones que, en numerosas ocasiones, repercuten en una ordenación del espacio que se pliega a las posibles fusiones de zonas funcionales. En este escenario, vemos que a menudo la existencia humana no puede más que adaptarse a las disposiciones espaciales que el objeto técnico sugiere por su propia estructura. 4) Así como el objeto técnico suele motivar la ordenación espaciante, el mismo también es centro de ocurrencia de causalidades físicas, las mismas imponen límites a las actividades del agente humano, quien debe acomodarse a dichos encadenamientos procesuales si desea que la actividad técnica sea realizada exitosamente. La materia trae sus exigencias y límites, ante ellos, la existencia humana no puede más que responder de forma adaptativa, una vez más, observamos que la centralidad del agente humano queda comprometida. 
5) por último, la perspectiva que prioriza la función a la hora de explicar el modo de ser del artefacto deriva del modelo hylemórfico de aplicación de forma a materia, este modelo es insuficiente para explicar la complejidad de la realidad técnica. No es el agente humano el que imprime una forma en una materia sino, propiamente, el conjunto técnico; el operario sólo acondiciona la materia y la forma para que el acomodamiento de las dos semicadenas ocurra. Asimismo, no cualquier forma se deja aplicar a cualquier materia, sino que en la adquisición de forma, deberán respetarse las potencialidades físicas del material. En todos estos aspectos, el ser humano no tiene la última palabra, sino un papel adaptativo. El resultado fue una imagen de la existencia humana como relativamente desplazada del centro de la escena técnica.

\section{Referencias bibliográficas}

Heidegger, M.: (1994) La pregunta por la técnica, en Conferencias y artículos, Barcelona: Ediciones de Serbal, Traducción de Eustaquio Barjau

(2000): Los problemas fundamentales de la fenomenología, Madrid:Trotta, Trad. y prólogo de Juan José García Norro

(2002): Interpretaciones fenomenológicas sobre Aristóteles, indicación de la situación hermenéutica [informe Natorp] Madrid: Trotta. Trad. de Jesús Adrián Escudero

(2004): Lógica: la pregunta por la verdad, Madrid: Alianza. Versión española de J. Alberto Ciria.

(2006) Ser y Tiempo, Madrid: Trotta. Traducción y notas de Jorge Eduardo Rivera

(2007) Prolegómenos para una historia del concepto de tiempo, Madrid: Alianza. Traducción de Jaime Aspiunza

(2013) Problemas fundamentales de filosofía antigua, Buenos Aires: Waldhuter

Husserl, E. (2008) La crisis de las ciencias europeas y la fenomenología trascendental, Prometeo: Buenos Aires.

Ihde, D. (2010) Heidegger's technologies, postphenomenological perspectives. New York: Fordham University Press

Parente, D. - Crelier, A.: (2015) la naturaleza de los artefactos: intenciones y funciones en la cultura material. Buenos Aires: Prometeo

Simondon, G. (2007) El modo de existencia de los objetos técnicos, Buenos Aires: Ed. Prometeo,Trad. Margarita Martinez y Pablo Rodriguez . 
(2009). La individuación. A la luz de las nociones de forma y de información. Buenos Aires: Cactus

Tugendhat, E.: (1970) Der Warheitsbegriff bei Husserl und Heidegger, Berlin: Walter de Gruyter \& Co. 
